Cahiers d'Études Germaniques

$77 \mid 2019$

Histoire des juifs et des juives d'Allemagne

(1867-1933)

\title{
„Diaspora“ im Werk jüdischer Autoren des Expressionismus
}

"Diaspora » dans la littérature expressionniste judéo-allemande

'Diaspora' in German-Jewish Expressionist Literature

\section{Katja Schubert}

\section{(2)enEdition}

Journals

Édition électronique

URL : https://journals.openedition.org/ceg/7172

DOI : $10.4000 /$ ceg. 7172

ISSN : 2605-8359

\section{Éditeur}

Presses Universitaires de Provence

\section{Édition imprimée}

Date de publication : 25 septembre 2019

Pagination : 93-105

ISBN : 979-10-320-0234-6

ISSN : 0751-4239

Référence électronique

Katja Schubert, „"Diaspora" im Werk jüdischer Autoren des Expressionismus", Cahiers d'Études Germaniques [Online], 77 | 2019, Online erschienen am: 25 März 2021, abgerufen am 15 Juni 2021. URL: http://journals.openedition.org/ceg/7172 ; DOI: https://doi.org/10.4000/ceg.7172 


\section{"Diaspora“ im Werk jüdischer Autoren des Expressionismus}

Katja SCHUBERT

Université Paris Nanterre / CEREG (EA 4223)

\section{Einleitung}

Die deutsch-jüdische Literatur wird in der Regel entlang der Alternative zwischen einem assimilativ-liberalen und einem dissimilativ-nationalistischen Kulturmodell beschrieben. Ernst Lissauer radikalisierte dies 1912 in der Formel, entweder auswandern; oder: deutsch werden' [...]. Historiographisch lässt sich diese freilich allzu schematische Alternative durch ein geschichtliches Narrativ differenzieren. Demnach wurden die Anfänge des jüdischen Schreibens in deutscher Sprache im späten 18. und frühen 19. Jahrhundert zuerst durch eine kulturelle Öffnung möglich:Auf der Basis der veränderten ideellen Bedingungen der Aufklärung sowie der rechtlichen, ökonomischen und politischen Bedingungen der Emanzipation konnten sich die bis ins 18. Jahrhundert weitgehend ausgegrenzten Juden zunehmend in die bürgerliche Gesellschaft integrieren. In dem Maße, wie dabei Bildung, Sprache und Kultur eine tragende Rolle spielten, lässt sich die deutschjüdische Literatur als das Produkt jener Liberalisierung und Kulturalisierung des Judentums an der Schwelle zur Moderne einordnen. ${ }^{1}$

Wenn man diese einleitenden Überlegungen aus Andreas B. Kilchers Text "Diasporakonzepte“ im Handbuch der deutsch-jüdischen Literatur weiter denkt, kommt man schnell zu der allgemein bekannten Feststellung, dass dieVersprechen von Emanzipation und Assimilation durch den wachsenden Antisemitismus im Verlauf des 19. Jahrhunderts nicht eingelöst werden. Die Schaffung einer neuhebräischen Kultur in Deutschland auf jüdischer Seite, auch in Verbindung mit dem Zionismus, ist eine Antwort auf das Scheitern der Gleichberechtigung und legt den Akzent der deutsch-jüdischen Bindestrich-Kultur nun in erster Linie auf das Jüdische und nicht mehr auf das Deutsche. Kilcher bricht diese Dichotomie auf und verweist auf ein drittes Narrativ,

1. Andreas B. Kilcher, "Diasporakonzepte”, in Hans Otto Horch (Hrsg.), Handbuch der deutschjüdischen Literatur, Berlin, de Gruyter, 2016, S. 135-150, hier S. 135. 
ein dezidiert diasporisches Modell, wonach die Jüdische Literatur und Kultur gerade nicht durch nationalkulturelle Zugehörigkeit bestimmt werden kann, [...] sondern wesentlich universal, kosmopolitisch, exterritorial oder transnational ist. ${ }^{2}$

Dieser Ansatz referiert auf ein vormodernes religiöses Diasporamodell, das auf Erfahrungen von Vertreibung, Verfolgung und Ausschluss basiert, um darin ein ästhetisch-politisches kreatives Potential auszumachen, das die jüdische Moderne begründet, die sich „weder über bürgerliche Integration noch über die Nationalstaatlichkeit, sondern über eine transnationale Literarizität definiert" ${ }^{\prime 3}$. Damit greift Kilcher die Idee der Substitution von Land und Synagoge durch das Buch auf und schreibt so die Reflexionen von Heine, Zunz und Steinschneider aus dem 19. Jahrhundert fort ins beginnende 20. Jahrhundert, wo die jüdische Diaspora nun noch einmal explizit ihr ganzes Vertrauen auf die metaphorische, literarische und exterritoriale Stärke des Buches setzt, und zwar von der Bibel bis zur Literatur generell. Dan Diner bestätigt diese Analyse und leitet daraus seine Arbeitsweise und Methodik für die von ihm herausgegebene und 2017 fertig gestellte Enzyklopädie jüdischer Geschichte und Kultur ${ }^{4}$ ab. Er definiert entsprechend die Bewegung der Moderne für Juden zwischen 1750 und 1950 in einem Dreierschritt, der vom Gesetz über die Geschichte, die von Yosef Hayim Yerushalmi als der "Glauben ungläubiger Juden" ${ }^{5}$ bezeichnet wird, hin zum Text verläuft.

ZuAnfang des20.Jahrhundertsfindet, unterdemEinflussderstärkerwerdenden zionistischen Diskurse, des Ersten Weltkriegs und der revolutionären Bewegungen am Ende des Krieges, eine weitere Differenzierung in den Diasporamodellen statt. Diese umfassen einen Diasporanationalismus Dubnowscher Provenien $z^{6}$, wonach eine jüdische Nation innerhalb der verschiedenen Nationalstaaten existiert, eine sozialrevolutionäre Form, in welcher die Revolution eine neue Gemeinschaft, unabhängig von der religiösen, ethnischen oder nationalen Zugehörigkeit schaffen soll oder auch ein eher bürgerlich-humanistisches Modell, das, so noch einmal Andreas Kilcher, unter anderem Feuchtwanger und Zweig vor allem im Exil vertreten. $^{7}$

Im Kontext der expressionistischen Dichtung im ersten Viertel des 20. Jahrhunderts eignen sich die jüdischen Autorinnen und Autoren diese Modelle ihrerseits auf verschiedene Weise an. Das jüdische Konzept des Exils wird von den jüdischen Expressionisten erstens verwendet, um zunächst „ihre doppelte Außenseiterexistenz als Juden und avantgardistische Intellektuelle und Künstler

2. Ibid., S. 136.

3. Ibid., S. 137.

4. Dan Diner, Enzyklopädie jüdischer Geschichte und Kultur, Stuttgart, Metzler, 2017.

5. Yosef Hayim Yerushalmi, Zachor: Erinnere dich! Jüdische Geschichte und jüdisches Gedächtnis, Berlin, Wagenbach, 1982, S. 92.

6. Siehe hierzu: Simon Dubnow, Die jüdische Geschichte. Ein geschichtsphilosophischer Versuch, dt. 1897; zweite Auflage: Frankfurt a. M., Kauffmann, 1921; Anke Hilbrenner, Diaspora-Nationalismus. Zur Geschichtskonstruktion Simon Dubnows, Göttingen, Vandenhoeck \& Ruprecht, 2007.

7. Kilcher, Diasporakonzepte, S. 141. 
zu begreifen" ${ }^{8}$. Exil wird also gedeutet als eine Erfahrung im Lande, in dem man geboren und aufgewachsen ist und wo der jüdische Künstler als progressiver, hellsichtiger Paria existiert. Ein Teil der jüdischen Poeten vertritt zweitens einen Diaspora-Begriff, „in dem das Exil eine geradezu utopische Funktion erhält, [...] nämlich als Programm und Realisation eines Kosmopolitismus, der mit Namen wie ,Europa', ,Welt' oder ,Erde' benannt wurde" ${ }^{\prime 9}$. Simultan dazu findet drittens eine Bewegung statt, die sich, auch beeinflusst durch Ideen der sozialistischen Revolution, vor allem in den Städten den Außenseitern und gesellschaftlich Erniedrigten zuwendet (Prostituierte, arme Trinkerkerle, traurige Poeten) und diese zu einer Chiffre für die exilische Existenz jüdischer Intellektueller im deutschsprachigen Raum transformiert. Interessanterweise beschreiben sich zur selben Zeit auch Intellektuelle nichtjüdischer Herkunft, die nach dem Krieg die Gesellschaft fundamental in Frage stellen, in ihrer Außenseiterposition mit Attributen, die aus jüdischen Schicksalen bekannt sind. Ein Beispiel ist Otto Flake 1920 im Neuen Merkur: „Wir Intellektuelle teilen im jetzigen Deutschland das Los der Juden, außerhalb zu stehen, geistig heimatlos zu sein, keine Gemeinschaft mit dem zu haben, was als nationales Denken gilt." ${ }^{10}$

Diese „Deutungen“ von Diaspora verquicken Exil, Utopie, Außenseitertum und avantgardistische Kunst, um schließlich die Figur des jüdischen Poeten an sich als Inbegriff der diasporischen Figur auszurufen, wie dies der expressionistische Dichter Alfred Wolfenstein 1921 formuliert: „Der Dichter ist der unter die Völker Verstreute; aus tieferem Grunde kommend und in höherem Sinne ortlos, der Verbannte. Er ist, zumal heute, der ungewiss wohnende unter Fremden." ${ }^{11}$ Man kann diese Figur des Zusammentreffens von Jude und Dichter viel später und nach der Shoah, im Jahre 1959, als vorangestelltes Zitat von Marina Zwetajewa „Bсе поэты жиды, Alle Dichter sind Juden“12 in dem Gedicht Und mit dem Buch aus Tarussa von Paul Clan wiederfinden.

\section{Sichtbare Unsichtbarkeit, Reihungsstil, Simultaneität}

Zur Arbeit als jüdischer Künstler in der Diaspora gehört auch in den expressionistischen Kreisen immer wieder die Frage, ob und wenn ja, in welchen Momenten der Existenz und der schöpferischen Tätigkeit man sich dezidiert als Jude versteht. Zuschreibungen von außen, Familientraditionen und Erfahrungswelten in bestimmten historischen Konfigurationen spielen eine

8. Hanni Mittelmann, „Jüdische Expressionisten: Identität im Aufbruch - Leben im ,Aufschub”“, in Hans Otto Horch, Charlotte Ward (Hrsg.), Jüdische Selbstwahrnehmung, Tübingen, Niemeyer, 1997, S. 181-194, hier S. 183.

9. Andreas B. Kilcher, "Physiognomik und Pathognomik der jüdischen Moderne. Anton Kuhs anarchistische Sendung des Judentums", Ashkenas - Zeitschrift für Geschichte und Kultur der Juden 10 (2), 2000, S. 361-388, hier S. 377.

10. Mittelmann, Jüdische Expressionisten, S. 183.

11. Ibid., S. 187.

12. Paul Celan, Die Niemandsrose, Frankfurt a. M., Fischer, 1985, S. 78. 
zentrale Rolle für eine sich im Laufe des Lebens auch wandelnde Selbstdefinition und Identifikation mit "dem Jüdischen" oder "dem jüdischen Volk".

Jakob van Hoddis, der mit seinem Gedicht Weltende aus dem Jahre 1911 den Auftakt zu einer tiefen Zäsur innerhalb der deutschsprachigen Literatur schafft, kommt 1887 in Berlin in einer assimilierten jüdischen Familie als Hans Davidsohn zu Welt. Den Geburtsnamen könnte man als eine Illustration der gewünschten deutsch-jüdischen Symbiose durch die Eltern lesen. Die sichtbare hebräische Genealogie möchte der Sohn zumindest teilweise auslöschen, indem er 1909 die anagrammatische Namensveränderung von Davidsohn zu van Hoddis vornimmt. Gleichzeitig aber ersetzt er den deutschen Vornamen Hans durch den hebräischen Vornamen Jakob. Der Germanist Theo Buck behauptet, dass der Namenswechsel die Distanznahme von seiner jüdischen Familie bedeutet. ${ }^{13}$ Man könnte dem entgegenhalten, dass sowohl durch den neuen Vornamen als auch durch das Anagramm der hebräische Name in gewisser Hinsicht noch ganz anwesend ist, allerdings in einer Form der Verborgenheit. „Das Jüdische“ würde sich demnach in einer Figur des Anwesend-Abwesenden manifestieren, gegen den Vaternamen gerichtet, dabei aber im Vornamen zurück greifend auf einen noch älteren Stammvater als David, nämlich Jakob. ${ }^{14}$

Van Hoddis schreibt im Oktober 1912 an seinen Freund Erwin Loewenson in Berlin einen Brief, der sich ebenfalls mit der Frage der Herkunft, dieses Mal der geistigen und religiösen, beschäftigt:

Mein Programm ist Krieg dem intellektuellen Antisemitismus und seiner Logos-Theorie. Krieg
der Aufklärung der Kabbala dem Talmud dem Hofmannsthal der protestantischen Mystik dem
Protestantismus [...] Im übrigen darf jeder glauben, was er will. Nur Geld muss ich haben. ${ }^{15}$

Interessant ist diese Rede in der Hinsicht, dass sie den Antisemitismus dem intellektuellen Diskurs zuschreibt in einer Zeit, in der die Dreyfus-Affäre, Pogrome in Osteuropa und erfahrener Antisemitismus im Alltag in der christlichen Mehrheitsgesellschaft längst zu jüdischen Lebenswelten gehören. Aufschlussreich ist auch, dass dieser intellektuelle Antisemitismus mit der griechischen Logos-Philosophie verbunden wird, was einen starken Kontrast bildet zu einem in der selben Zeit schreibenden Hermann Cohen, der im Logos des Griechentums einenverbindendenUrsprungzwischen Judenund Christen sieht. ${ }^{16}$ Diekommalose Rede, verfasst im Ton der Kriegserklärung zwei Jahre vor dem Beginn des Ersten Weltkriegs, ist aber auch vielsagend, weil sie eine aggressive programmatische Nichtzugehörigkeit im scheinbar spielerischen Nebeneinander und in der

13. Theo Buck, Streifzüge durch die Poesie. Von Klopstock bis Celan. Gedichte und Interpretationen, Köln/ Wien/ Weimar, Böhlau, 2010, S. 191.

14. Dass van Hoddis Jude war und 1942 in Sobibor ermordet wurde, wurde noch bis in die 1990er Jahre an deutschen Gymnasien und in Germanistikseminaren an den Universitäten nur selten angesprochen.

15. Regina Nörtemann (Hrsg.), Jakob van Hoddis: Dichtungen und Briefe, Göttingen, Wallstein, 2007, S. 191.

16. Herman Cohen, Deutschtum und Judentum (1915), in Werke, 16 Bde., Hildesheim, Olms, 1978-1997, Bd. 16. 
Gleichzeitigkeit von geistig-religiösen-politischen Strömungen propagiert. Man findet eine solche Form wieder in van Hoddis' Gedichten, die maßgeblich den berühmt gewordenen expressionistischen Reihungsstil der Simultaneität, der die Gleichzeitigkeit von Geschehen ausdrücken will, geprägt haben. Der Reihungsstil stellt den Versuch dar, ein kompaktes Schöpfungsmodell der Minimalisierung in der Bändigung des Materials zu produzieren, das als Inventar, Resümee oder Erinnerungsspeicher dienen kann. Die rigorose Stakkatoform erzeugt klanglichrhythmische Muster und verbindet sie oftmals mit sehr heterogenen Inhalten und der Aneinanderreihung von Bildern (Metaphern), die nicht zwingend in direktem (syntaktischen und logischen) Zusammenhang stehen und durch die meist verschiedene Sinneseindrücke sequenzartig wiedergegeben werden. Ordnung und Unordnung zugleich strahlen davon aus. Jüdische und nichtjüdische Künstler treffen sich in dieser Praxis einer neuen Ästhetik, in welcher die eigene religiöse oder ethnische Herkunft keine Rolle spielt. Und doch ist auffällig, dass van Hoddis in dieser formalen Revolte immer wieder auf "das Jüdische" zurück kommt. In einem langen Brief an Loewenson aus dem Jahre 1913 begründet er seine Konversion zum Katholizismus. Der Schlussteil des Briefes enthält dann noch folgende Anmerkungen: „An das Volk der Juden glaube ich noch immer [...] Das Kaffeehaus ist von Idioten bevölkert. Ich möchte gerne eine Zeitschrift haben um ein katholisches Judentum zu gründen.." ${ }^{17}$ Sechs Wochen später schreibt er an den selben Freund: "Mein Judentum (übrigens) bricht wieder durch. Der Katholizismus war nur ein Abenteuer." ${ }^{18}$ Von diesem Durchbruch ist explizit oder als bekennendes Prinzip wenig die Rede im nachfolgenden Werk. Man versteht, dass es um einen "reinen Durchbruch" oder eine bindende Gemeinschaft auch gar nicht gehen kann oder soll. Eine Haltung des Experimentieren und der Distanzierung, die das Risiko der Selbstauflösung mit einbezieht, verwahrt sich im Fall dieses Dichters gegen jede Vereinnahmung und Instrumentalisierung. Das schließt allerdings die Bezugnahme auf gewisse literarische Traditionen nicht aus. Tatsächlich bezieht sich die Simultaneität bei van Hoddis auch auf poetische Prinzipien, die „bis zu Homer reichen“" ${ }^{19}$, der als Gewährsmann für die Unabhängigkeit von Zeit und Zeitbedingtheit angeführt wird, um in der Literatur eine "unermessliche Weltweite ${ }^{\prime 20}$ zu erzeugen. Wenn Homer eine Schlacht schildere und das Waffengeklirr mit dem Schlag des Holzfällers vergleiche, wolle er zeigen, dass während der Schlacht gleichzeitig auch Waldesstille sei, erschüttert vom Schlag der Holzfäller. ${ }^{21}$ Ein anderes griechisches Erbe also als das der Logostheorie, nämlich das epische Erzählen, wird hier herangezogen, auch mit dem Potential leiser Töne im Gegenüber zum expressionistischen Aufruhr.

Wird in der Sekundärliteratur die oftmals zerreißende, überfordernde Simultaneität schon immer auch mit der veränderten Wahrnehmungsweise

17. Nörtemann, van Hoddis, S. $193 f$.

18. Ibid., S.195.

19. Ibid., S. 311.

20. Ibid., S. 310.

21. Ibid. 
durch das moderne Großstadtleben erklärt, so könnte man darin auch eine spezifisch jüdisch-diasporische Lebenskonfiguration der Epoche erkennen: offiziell gleichberechtigte Staatsbürger seit der Verfassung von 1871 und als Bürgertum zu Wohlstand gekommen, bleiben Juden ausgeschlossen von höheren Ämtern in Armee, Universität, Bürokratie und Politik. Neben der rechtlichen Gleichberechtigung bedroht der moderne, auf Rassentheorien basierende Antisemitismus Juden im Alltag. Gleichzeitig konsolidieren sich säkulare Formen jüdischer Kultur, aber Juden werden nun weniger für ihren Aberglauben, dafür aber umso mehr für ihre Verstandesreligion und ihre Urbanität kritisiert. Die Widersprüche und Aporien dieser Konstellation und die äußerst ambivalenten Botschaften, die von der christlichen Mehrheitsgesellschaft ausgesendet werden, beschreiben ihrerseits eine merkwürdige Simultaneität offener und verschlossener Räume. Jüdische expressionistische Dichter, so meine These, versuchen auch deshalb, den Anteil des „Offenen“ darin immer wieder zu erweitern, indem sie simultan und nicht selten "in Reihung" sachlich-analytisch, visionär-utopisch, grotesk, ernsthaft-pathetisch oder surreal schreiben. Diesen Stimmenrausch könnte man ebenfalls mit dem diasporischen Geist des Universalen, des Kosmopolitischen und des Exterritorial-Transnationalen assoziieren. Heraus kommen kann dabei ein Visionarr, nach einem Titel eines Gedichts von van Hoddis aus dem Jahre 1914, worin Eros, Leben, Sterben und Töten nur durch eine hauchdünne Wand getrennt sind:

Aus der Wand fuhr ein dünner Frauenarm.

Er war bleich und blau geädert.

Die Finger waren mit kostbaren Ringen bepatzt.

Als ich die Hand küsste, erschrak ich:

Sie war lebendig und warm.

Das Gesicht wurde mir zerkratzt.

Ich nahm ein Küchenmesser und zerschnitt ein paar Adern. ${ }^{22}$

\section{Volk, antinationale Moderne und Revolution}

Am Werk des 1886 in einem Wiener Arbeiterviertel geborenen Dichters und Journalisten Albert Ehrenstein kann man auf exemplarische Weise das Schwanken und Suchen, die verschiedenen Aneignungen und Abstoßungen eines fast lebenslang diasporischen Schreibens aufzeigen. Anders als van Hoddis wird Ehrenstein in verschiedenen Texten ab 1917 sehr explizit und "unverborgen" als Jude schreiben und zugleich Diaspora-Modelle experimentieren, in dem „das Jüdische" eine Art der Aufhebung erfahren soll. Auf Anraten seines Förderers Karl Kraus siedelt Ehrenstein 1911 in die deutsche Kulturmetropole Berlin über, die Stadt, der er zeitlebens verbunden bleibt. Er gehört zur Generation der nicht nur expressionistischen und nicht nur jüdischen Künstler, die von einer patriotisch-chauvinistischen Begeisterung am Anfang des Krieges erfasst 
werden. So rezensiert er 1914 ein Werk über neuste Kriegslyrik, worin Dichter wie Hauptmann oder Dehmel, seiner Meinung nach, „endlich ganz zum Volk heimgefunden" ${ }^{23}$ haben und „wackere, fromme, mundgerechte und vor allem verständliche Kriegslieder dem Soldaten“24 geben. Laut Hanni Mittelmann verbirgt sich hinter diesem verklärten „Krieg als Vater aller Dinge“ ein Sehnen nach dem Erlebnis der Gemeinschaft, "das die Isolation des Künstlers innerhalb des ,Volkes' zu überwinden schien“25. Man kann hier ergänzen, dass diese Sehnsucht nach Gemeinschaft wohl auch im Zusammenhang mit der Position als Jude steht, so wie ein Viktor Klemperer, trotz der Möglichkeit einer Freistellung, in den Krieg für Deutschland zieht und Hermann Cohen die jüdischen Soldaten als Zeichen der Integration von Juden in die deutsche Gesellschaft interpretiert, bevor 1916 durch die Judenzählung mit antisemitischem Hintergrund genau diese erträumte Gemeinschaft fundamental in Zweifel gezogen wird. Im ersten Kriegsjahr findet sich also bei Ehrenstein noch keine Spur von diasporischem Schreiben oder von Suche nach jüdischer Gemeinschaft, wie sie Michael Brenner für viele Juden in der Weimarer Republik beobachtet. ${ }^{26}$ Das Schreiben bezeugt viel eher eine Anziehung durch nationale Ideen und rekurriert auf das Bild eines deutschen Volkes, dem Juden ohne Frage angehören bzw. worin Juden vielleicht die Hoffnung haben, wie die Dichterstimmen, über die Ehrenstein schreibt „"ganz zum Volk heimzufinden".

EinegroßeVeränderungkannmandreiJahrespäterineinerKindheitserinnerung in seinem Text Menschlichkeit aus dem Jahre 1917 konstatieren. Ehrenstein spricht nun explizit als Jude, ganz als ob der Krieg zur Formierung dieses Selbstverständnisses beigetragen habe. Das geschah im übrigen auch durch die Begegnung jüdischer Soldaten mit Ostjuden bzw. durch die 70000 Ostjuden, die zwischen 1916 und 1920 nach Deutschland kamen, wobei Idealisierung, Identifikation und Abstoß gleichermaßen als Reaktion zu verzeichnen sind. ${ }^{27}$ Ehrenstein beginnt seine Erinnerung an die Zeit als junger österreichischer Vorstadtjude mit dem bitteren Beschimpft-Werden als "Saujud" durch die Kameraden auf dem Schulweg. Dem folgen Bilder zum Erleben der jüdischen Religion: Einerseits ist er "dem Gebet frommer Großeltern gehorsam “28, was wohl auch impliziert, dass es die Eltern nicht mehr sind und ein durch die Säkularisierung bedingter Generationenwandel stattgefunden hat. Andererseits ist es genau der religiöse Ritus, der sich ihm selbst auch bereits in der Tiefe entzieht, sich ihm allenfalls noch in einer sinnlichen und gefühlsbeladenen Ästhetik erschließt, aber auch schon mit dem analysierenden Blick auf eine der Religionsvermittlung inhärente Gewalt der Vereinnahmung und Fremdbestimmung verbunden wird:

23. Hanni Mittelmann (Hrsg.), Albert Ehrenstein: Neueste Kriegslyrik, in Albert Ehrenstein, Werke. Bd. 5, Aufsätze und Essays, Göttingen, Wallstein, 2004, S. 66.

24. Ibid.

25. Ibid., S. 575 .

26. Michael Brenner, Jüdische Kultur in der Weimarer Republik, München, C. H. Beck, 2000, S. 19.

27. Ibid., S. 44.

28. Mittelmann, Ehrenstein, S. 296. 
Das Judentum selbst sprach zu mir mit heiligen Klängen, die ich kaum verstand, ergreifenden Zeremonien, die mich in Gebetmänteln umrauschten, mit Riemen banden. Die Schönheit des Seders ließ mich aber keineswegs die blutige Schattenseite: Beschneider und Schächter, vergessen, noch gar die pharisäischen ,Religionslehrer' und Rabbiner: Schächter, die den kindlichen Glauben beschnitten. ${ }^{29}$

Die Auflehnung gegen die Autorität der Väter, ein Leitmotiv der gesamten expressionistischen Dichtung, wird durch eine beißende "jüdische" Sozialund Kulturkritik noch zugespitzt, da diese jüdischen Väter und Lehrer mit oberflächlichem Halbwissen operierten, das sie überdies lieber an wohlhabende als an arme Judenkinder weitergäben und zwar interessanterweise deshalb, weil gerade diese ärmeren „jenes hebräische Wissen besaßen, das die Lehrer selbst nicht vermittelten“"30. Genau dieses Wissen stellt Ehrenstein abfällig den guten Noten der reicheren Kinder entgegen,

nur weil sie jüdische Geschichte, historischen Schwafel phrasenreich wiedergeben konnten - hie und da schwamm dann in die Sprechstunde des darob brillantinebeglückten Professors ein edelsteinbehangenes, seidenrauschendes Häufchen Gänseschmalz, die Frau Kommerzialrat. ${ }^{31}$

In diesen wenigen Sätzen Ehrensteins bietet sich ein sozial und kulturell vielfältiges Bild jüdischer Lebenswelten zu Beginn des 20. Jahrhunderts in den europäischen Städten dar: die Herkunft aus dem Arbeitervorort oder aus der bürgerlichen Schicht, die Kinder der religiösen Ostjuden, die in Ehrensteins Perspektive arm sind, aber ein „authentisches" Wissen verkörpern und hebräisch können, was der Krise von Religion und Glaube und der Tatsache der "verborgenen Tradition", wie Hannah Arendt das im anderen Zusammenhang nennt ${ }^{32}$, im assimilierten, ins Bürgertum aufgestiegene Judentum gegenübergestellt wird. „Assimilanten“ nennt Ehrenstein die in diesem Sinne Erfolgreichen, die sich in, wenn auch ebenfalls schlecht gelehrter, jüdischer Wissenschaft und vor allem Geschichte mehr wiedererkannten als im alten Ritus. ${ }^{33}$ Das von Ehrenstein illustrierte Bürgertum scheint allerdings eher mit der gesellschaftlichen Anpassung und dem Aufstieg beschäftigt zu sein als mit der Hinwendung zu dem alt-neuen kulturellen und spirituellen Zentrum des Judentums, zu Buch und Text. Sehr klarsichtig im Hinblick auf diese Gruppe folgt dann auch seine Bemerkung, dass dieser Weg in die Moderne, mit oder ohne vertieftes jüdisches und nicht jüdisches Buchwissen, aufs höchste gefährdet sei und die Juden möglicherweise nicht vor Antisemitismus, d. h. Mord, schützen werde. Denn genau diese bürgerliche Schicht „mit Monokel und Eisernem Kreuz [...], (die) früh anhob mit den Worten,Gestern hab ich den ganzen Nietzsche geschenkt gekriegt ${ }^{\prime \prime \prime}{ }^{34}$ werde „am Pogromtag unter dem losgelassen ,Dö Juden san schuld' brüllenden Hunger" ${ }^{\prime 35}$ ihr Ende finden.

\footnotetext{
29. Ibid., S. 297.

30. Ibid.

31. Ibid.

32. Hannah Arendt, Die verborgene Tradition. Essays, Frankfurt a. M., Suhrkamp, 1976.

33. Mittelmann, Ehrenstein, S. 297.

34. Ibid., S. 297.

35. Ibid.
} 
Eine andere, sehr politisierte Antwort auf diese falsche Illusion der assimilierten Juden geben Ehrenstein, Franz Pfemfert, Carl Zuckmayer und andere Künstler 1918 im ersten Heft der Aktion nach dem Revolutionsausbruch im November, worin sie den Aufruf der Antinationalen Sozialisten Partei, Gruppe Deutschland (A.S.P.) verfassen. Vom im Krieg abgeschlachteten werktätigen Volk ist die Rede, vom Marsch der sozialistischen Revolution, von den „intellektuellen Haßsängern“ und den gewerbsmäßigen „Führern“ und von der völkerexpropriierenden Diktatur des Kapitalismus. ${ }^{36}$ Das Volk ist nun nicht mehr das völkische Deutschland, wie es Ehrenstein noch 1914 herbei phantasiert hat, es ist auch kein jüdisches Volk, sondern es ist das internationale Volk der Arbeiter, der Brüder, seien sie serbisch, italienisch, französisch, russisch oder englisch, Ost und West nunmehr vereint, im Gegensatz zur Spannung zwischen den zuvor genannten ost- und westjüdischen Schülern. Es ist das Volk in einer internationalen Dimension, das Opfer, Akteur, Kämpfer und Revolutionär in einem ist: „Nieder mit den Vaterländern“, „es lebe das grenzpfahllose Land der arbeitenden Menschheit" ${ }^{\prime 37}$, lauten die Parolen. Die „Jüdische Frage" wird hier im transnationalen Paradigma der proletarischen kämpferischen Brüderlichkeit und der von ihr getragenen Revolution aufgehoben, also nicht mehr explizit genannt.

Und doch kommt die Auseinandersetzung damit auf einem anderen Weg zurück, und zwar in Ehrensteins Ausführungen zum seiner Meinung nach reaktionären und anachronistischen Zionismus. Ehrenstein sieht in der Errichtung eines eigenen Staats für Juden keine Erfüllung, sondern bezeichnet dieses Ansinnen provokativ in einem Essay mit dem Titel Nationaljudentum als "Kreuzzug" und als "Heimweh nach einem noch größeren Ghetto" ${ }^{38}$, also in gewissem Sinne als eine dumpfe Umkehrung oder Replik der christlichen Geschichte und ihrer diskriminierenden Einrichtungen für Juden:

\footnotetext{
Ein jüdischer Nationalpark, ein Indianerterritorium, eine Reservation, in der statt wilder Bisons gemäßigte Israeliten verwahrt werden [...] das wäre Flucht, Flucht ins Herbarium. Und neue freiwillige Kasernierung, Uniformierung des Judentums [...]. Aber den Juden asiatisch neutralisieren, hieße, wichtigste Blutkörperchen, adlerstarken Sprengstoff der europäischen Menschheit entziehen. Es gibt eine höhere Dienstpflicht als die allgemeine, nationale, konfessionelle, wirtschaftliche [...] Deswegen möchte, aller Leiden ungeachtet, ich den Juden, einen der besten Streiter Gottes und der Menschheit, aus dieser aufreibenden Dienstpflicht noch nicht in den vegetativen Ruhestand, in den nabelbeschaulichen, lebensfristenden Ackerbau entlassen, auf Milch- und Honigflüssen dahintreiben. ${ }^{39}$
}

In versöhnlich-humorvollem Ton in der zweiten Zitathälfte hat hier nun die europäische Menschheit den Platz des internationalen Volks der Arbeiter eingenommen, und der Autor plädiert dafür, dass das christlich geprägte Europa, wenn nicht gar die Welt, die jüdische Diaspora als Lebenselixier braucht, und dies nicht zuletzt wegen der Literatur:

36. Ibid., S. $108 f$.

37. Ibid., S. 111.

38. Ibid., S. 309.

39. Ibid., S. $298 f$. 
In aller Jüdischen Dichtung finde ich ein Plus an Moral und Ethik, oft sorgfältig verborgen hinter einer übertrieben-zynisch witzelnden Maske. Der Stamm des alten Propheten lebt noch. Altes und Neues Testament sehnen sich nach einer Ergänzung, nach einem tröstlichen Ende. ${ }^{40}$

Ehrenstein legt hier eine ungewöhnliche Deutung der jüdischen Diaspora vor, indem ausgerechnet sie die Überwindung der Spaltung von Judentum und Christenheit, beide unerlöst, unvollendet und konfliktreich aufeinander bezogen, bewerkstelligen könnte. Anstelle des politischen Kampfes tritt das Bedürfnis nach Trost, ein neuer Ton mit religiösen Konnotationen wird angeschlagen.

\section{Juden, Israeliten, Hebräer, Jerusalemiten}

Zu Ehrensteins religiösem Timbre des Trosts passt, dass er die Bezeichnung „Israeliten“ im vorherigen Zitat gewählt hat. Im Zionismus werde der Jude zum Israeliten, werde er also wieder auf eine religiöse Zugehörigkeit beschränkt. Tatsächlich entspricht dies aber nicht der Realität des zionistischen Diskurses, der sich als säkulare Bewegung von Juden versteht. Else Lasker-Schüler, expressionistische Dichterin und Leserin von Ehrenstein und van Hoddis, spricht in ihren Gedichten von den Hebräern und vom Hebräerland, eine dritte Variante. Sie stellt den Hebräern die verweichlichten geschwächten Juden der europäischen Gegenwart gegenüber, der sie sich zunehmend entfremdet, nachzulesen unter anderem im 1905 publizierten Gedicht "Mein Volk", dessen erster Vers lautet: „Der Fels wird morsch, dem ich entspringe ${ }^{41}$. Für diese Autorin sind Emanzipation und Assimilation der Juden schon früh und definitiv gescheitert, und im Antisemitismus sieht sie "das Verhängnis nicht nur ihres eigenen Lebens und der deutschen Judenheit, sondern des Abendlandes überhaupt ${ }^{\prime 42}$. Sie verbindet den Begriff "Hebräer" einerseits stärker mit der Sprache und bezieht sich auf die ursprünglich in hebräischer Sprache überlieferten Bücher der Bibel, die vom Judentum als einzige zum Kanon der biblischen Bücher gerechnet werden. Alice Jacob-Loewenson, eine namhafte Musikkritikerin, veröffentlichte am 22. September 1922 in der Jüdischen Rundschau einige Übersetzungen von Gedichten Else Lasker-Schülers ins Hebräische. In ihrer Vorbemerkung schreibt sie:

Diese Dichtungen eignen sich besonders deshalb für eine solche Übertragung, weil ihre sprachlichen Mittel merkwürdig hebräisch anmuten; fast scheint es Zufall, dass nicht das Hebräische ihre Ursprungssprache ist. Ja, man könnte fast von einem hebräischen Deutsch sprechen. ${ }^{43}$

40. Ibid., S. 308.

41. Karl Jürgen Skrodzki (Hrsg.), Else Lasker-Schüler: Sämtliche Gedichte, Frankfurt a. M., Suhrkamp/ Insel, 2004, S. 93.

42. Alfred Bodenheimer, "Else Lasker-Schüler", in Andreas B. Kilcher (Hrsg.), Lexikon der deutschjüdischen Literatur, Stuttgart, Metzler, 2000, S. 375.

43. Karl Jürgen Skrodzki (Homepage), Else Lasker-Schüler: Mein Volk, [https://www.kj-skrodzki.de/ Dokumente/Text_012.html, Stand: 2. Februar 2019. Eine Erinnerung an die Buber-RosenzweigBibel-Übersetzung liegt hier nahe. 
Zugleich assoziiert Else Lasker-Schüler das Hebräische mit einer biblischen Erlösungssehnsucht des heiligen Gottesvolkes und mit Heimweh-Bildern einer idealisierten archaischen Landschaft und Lebenskultur im Grenzland von Diesseits und Jenseits, in welches sie auch Elemente der eigenen Kindheit einfügt. Die Hebräer sind für Lasker-Schüler aber auch die Antipoden der Zionisten, die sie im Peter-Hille-Buch „Jerusalemiter" nennt und wie folgt beschreibt: „Sie nahmen heimlich ihre Harfen und spielten darauf Misstöne statt der Lieder lieblicher Zebaothländer. ${ }^{\prime 4}$ Klingt hier nicht fast ein Verrat an einer bestimmten Form von Exil durch die Zionisten durch, wenn wir als Referenz den Psalm 137 erinnern: "An den Wassern zu Babel saßen wir und weinten, wenn wir an Zion gedachten. Unsere Harfen hingen wir an die Weiden ${ }^{\prime 45}$, was gemeint ist als Widerstand gegen die Herren im Exilland, die ein solches Lied als Demütigung der Gefangenen hören wollen?. Die Jerusalemiter haben die Harfen wieder von den Weiden abgenommen und singen, weil sie heimgekehrt sind. Doch die Töne, die sie produzieren, vielleicht jüdische nationale Lieder und Lyrik, können nur Misstöne sein. Das bestätigt auch der Plural - die Lieder "der Zebaothländer" -, der sich nicht mit der Idee des einen jüdischen Nationalstaats, wie sie der Zionismus vertritt, deckt.

Lasker-Schüler favorisiert hier ein diasporisches, jedoch sehnsüchtig auf Jerusalem ausgerichtetes und sich im Kontext der Jüdischen Tradition bewegendes Dichtungsmodell, das jüdische Dichtung in mehreren Ländern oder Nationalstaaten verortet und keine reale territoriale Verortung anstrebt. ${ }^{46}$

In der Tat interessiert das "reale Israel“ die Schreibende kaum, und ihre letzten Exiljahre in Israel, ein Land, in dem sie keine letzte Heimat finden konnte, bestätigen das.

\section{Ahasver}

Juden, Israeliten, Hebräer, Jerusalemiter - all diese Namen sind, und sei es ex negativo, auf ein Land bezogen, dessen historische Wurzeln in der Torah festgeschrieben wurden. Mit dem simultan stärker werdenden Zionismus und dem wachsenden Antisemitismus im völkischen Gedankengut belebt Ehrenstein noch eine andere Figur, um vom Schicksal der Juden in einer nicht nationalen Optik zu sprechen: Ahasver. Er kehrt die traditionelle, in einem antijüdisch-christlichen Kontext entstandene Legende, die als Basis der christlichen Schuldtheologie diente, um: Ahasver wird nun „zu einem ewigen Zeugen der Verfolgung der Juden

44. Else Lasker-Schüler, Werke und Briefe. Kritische Ausgabe. Bd. 5: Prosa, bearbeitet von Karl Jürgen Skrodzki und Itta Shedletzky, Frankfurt a. M., Suhrkamp/ Jüdischer Verlag, 2002, "Das Hebräerland“, S. 57.

45. Die Bibel, Übersetzung Martin Luther, Jubiläumsbibel, Stuttgart, Deutsche Bibelgesellschaft, 1981: Psalm 137, Verse 1 und 2.

46. Birgit M. Körner, Hebräische Avantgarde. Else Lasker-Schülers Poetologie im Kontext des Kulturzionismus, Köln/ Wien/ Weimar, Böhlau, 2017, S. 198. 
durch die Christen" 47 einerseits und andererseits zum Reisenden und ebenfalls zum Inbegriff des sich überall engagierenden Weltbürgers: "Gewiss ist der Ahasverismus, die Heimatlosigkeit ein bitteres Schicksal dem Einzelnen, aber sie ist ein Grund der verhältnismäßig großen Leistungen vieler Juden; zwingt sie in jedem Land in den Wetteifer mit jedem Volk." ${ }^{48}$ Mit der Verschiebung von Ahasver zum Begriff Ahasverismus bei Ehrenstein kann die Freiheit zur Kreativität und die Möglichkeit zur Selbst- und Neubestimmung integriert werden, die erneut den Künstler mit einbezieht. Letztlich sei das Bekenntnis zum Ahasverismus, so Ehrenstein, der Kern der Lehre aus der Jüdischen Geschichte: „nur ein unsichtbarer Tempel kann nicht zerstört werden " ${ }^{49}$, womit wir wieder in der Welt des Geistigen, des gelesenen Textes angelangt sind, der in den Menschen arbeitet und sie verbindet zur Gemeinschaft. Wolfenstein spricht von der "vornehmen Ruhelosigkeit" des Ahasver ${ }^{50}$ auch in der Absicht, die jüdische Intellektualität, die auch als antisemitisches Stereotyp zunehmend Verbreitung findet, positiv umzudeuten. Für Ehrensteins Freund, den jüdischen Dichter Ernst Weiss, gleicht Ahasver dann wieder eher dem Sozialrevolutionär, der "am tiefsten Ghettoluft ein- und ausatmet ${ }^{\prime 51}$, wenn er durch die exterritorialen Räume wie Hinterhöfe, Mietskasernen und Vororte streift, die zugleich aber Zentrum des Territoriums der Erniedrigten sind. Ein solcher ahasverischer Held ist der Protagonist der gleichnamigen Erzählung Tubutsch ${ }^{52}$ aus dem Jahre 1911, mit der Ehrenstein zu einem der Wortführer des Expressionismus wird. Ernst Weiss sieht in Tubutsch/ Ahasver darüber hinaus erneut eine Variante der Begegnung von Jüdischem und Griechischem, die nicht auf den Logos zentriert ist, sondern Ahasver als Bruder von Odysseus entwirft.

Doch die Ahasver-Figur nimmt ein paar Jahre später noch eine andere, verzweifeltere, hoffnungslosere Bedeutung an. In Ehrensteins Buch Briefe an Gott ${ }^{53}$ aus dem Jahre 1922 wird der "ewige Fremdling, der in der Luft schwebt wie die Sterne über der Wüste" ${ }^{45}$ zu einem Rufer in der leeren Wüste, da kein Gott und kein Mensch antworten. Das Leiden an der Leere und Sinnlosigkeit der Zeit, auch inspiriert vom Scheitern der Revolution 1918-19 und dem Fortbestehen von Nationalismus, Ungerechtigkeit und Antisemitismus, bekommt als individuelles und kollektives Erleben in Ahasver Gestalt. Diaspora ist nun nicht mehr an ein Terrain, auch nicht an ein inneres, geistiges, textuelles gebunden, es geht nicht mehr um wandern oder "im Fremden wohnen", auch nicht mehr um eine punktuelle Mission des Ahasver, sondern um ein Schweben, fast als ob hier

47. Armin A. Wallas, „Albert Ehrenstein“, in Andreas B. Kilcher (Hrsg.), Lexikon der deutsch-jüdischen Literatur, Stuttgart, Metzler, 2000, S. 128.

48. Mittelmann, Ehrenstein, S. 311.

49. Ibid., S. 312

50. Zitiert bei Mittelmann, Jüdische Expressionisten, S. 188.

51. Ernst Weiss, Die Ruhe in der Kunst. Ausgewählte Essays, Literaturkritiken und Selbstzeugnisse, 1918-1940, Berlin/Weimar, Aufbau, 1987. Zitiert bei [http://gutenberg.spiegel.de/buch/ essays-4416/9], Stand: 2. Februar 2019.

52. Albert Ehrenstein, Tubutsch, Wien, Jahoda \& Siegel, 1911.

53. Albert Ehrenstein, Briefe an Gott, Leipzig, Waldheim-Verlag, 1922.

54. Wallas, Ehrenstein, S. 129. 
der Luftmensch spricht. Es ist bekannt, dass dieser Begriff im antisemitischen Jargon zu jener Zeit zunehmend zum Inbegriff für die sogenannten „bodenlosen, unproduktiven" Juden wurde und dass die antisemitische Instrumentalisierung des Begriffs zu der Politik beitrug, die nach 1933 das europäische Judentum in der Tat vollkommen entwurzeln sollte. Es scheint, als ob Ehrenstein 1922, nachdem er fast alle Optionen diasporischen Schreibens durchlaufen hat und eine nach der anderen in gewisser Weise fallen ließ, auch weil die Geschichte die Probe aufs Exempel nicht bestanden hat, nun allein noch diesen Schwebezustand beschwören kann. 
